\title{
Anti-VEGF Therapy in Breast and Lung Mouse Models of Cancers
}

\author{
Di Domenico Marina, ${ }^{1}$ Ricciardi Carmela, ${ }^{1}$ Fusco Alfredo, ${ }^{2}$ and Pierantoni Giovanna Maria ${ }^{2}$ \\ ${ }^{1}$ Dipartimento di Patologia Generale, Seconda Università degli Studi di Napoli, Via L. De Crecchio, 7, 80138 Napoli, Italy \\ ${ }^{2}$ Dipartimento di Biologia e Patologia Cellulare e Molecolare, Istituto di Endocrinologia ed Oncologia Sperimentale del CNR c/o, \\ Facoltà di Medicina e Chirurgia di Napoli, Università degli Studi di Napoli "Federico II", Via Pansini 5, 80131 Napoli, Italy
}

Correspondence should be addressed to Di Domenico Marina, marina.didomenico@unina2.it

Received 20 September 2010; Accepted 1 October 2010

Academic Editor: Monica Fedele

Copyright ( $) 2011$ Di Domenico Marina et al. This is an open access article distributed under the Creative Commons Attribution License, which permits unrestricted use, distribution, and reproduction in any medium, provided the original work is properly cited.

\begin{abstract}
Cancer is the second leading cause of death in the world after cardiovascular diseases. Some types of cancer cells often travel to other parts of the body through blood circulation or lymph vessels, where they begin to grow. This process is recognized as metastasis. Angiogenesis is the formation of new blood vessels from existing vessel. Normally angiogenesis is a healthy process, that helps the body to heal wounds and repair damaged body tissues, whereas in cancerous condition this process supports new blood vessels formation that provide a tumor with its own blood supply, nutrients and allow it to grow. The most important proximal factor for angiogenesis is the vascular endothelial growth factor VEGF. Angioinhibition is a form of targeted therapy that uses drugs to stop tumors from making new blood vessels. Therefore, in this paper we analyse the importance of VEGF as target of cancer therapy, analysing murine models.
\end{abstract}

\section{Introduction}

Angiogenesis, the process by which the existing vascular network expands to form new blood vessels, is required for the growth of solid tumors [1]. For this reason, tumor angiogenesis has become a critical target for cancer therapy. Vascular endothelial growth factor (VEGF), a primary stimulant of angiogenesis, binds and activates VEGF receptor 1 (VEGFR1) and VEGFR2 [2]. VEGF is an important and powerful factor increasing vascular permeability and promoting metastasis. Without blood vessels, the tumors can not be larger than a few millimeters, so the inhibition of angiogenesis with the use of several drugs could represent an important tool in cancer treatment for several reasons. (1) Angiogenesis occurs at high levels during fetal development, the menstrual cycle, and in wound healing. Therefore, the treatments should have low toxicity; in fact, they might be expected to interfere with this process and should not harm most normal dividing cells. (2) The antiangiogenic treatments should not be designed to attack directly the cancer cells. The targets of several of these treatments are normal processes controlled by normal cells and not by the tumor cells themselves. The high mutation rates of cancer cells that often render chemotherapy ineffective will not interfere with these drugs.

In this paper, we underline the importance of inhibition of VEGF as attractive therapeutic target in the treatment of cancer. VEGF is a primary stimulant for tumor angiogenesis, making it a critical target for cancer therapy $[3,4]$. In breast cancer, elevated levels of VEGF correlate with increased lymph node metastases and a worse prognosis [5]. Actually, bevacizumab, a humanized monoclonal antibody that binds human VEGF and prevents VEGF from binding VEGFR1 and VEGFR2, is approved for the treatment of metastatic HER2/NEU-negative breast cancer [6].

\section{VEGF and Breast Cancer}

VEGF is a primary stimulant of angiogenesis and is a macrophage chemotactic protein [7]. Inhibition of VEGF is beneficial in combination with chemotherapy for some breast cancer patients. Anti-VEGF therapy with bevacizumab, the phenethylamine of the $2 \mathrm{C}$ family $2 \mathrm{C} 3$ or the fully human antibody that inhibits VEGF binding to VEGFR2 r84 inhibits the growth of established orthotopic MDA-MB-231 breast cancer cell line in severe combined immunodeficiency (SCID) mice [8], reduces tumor 


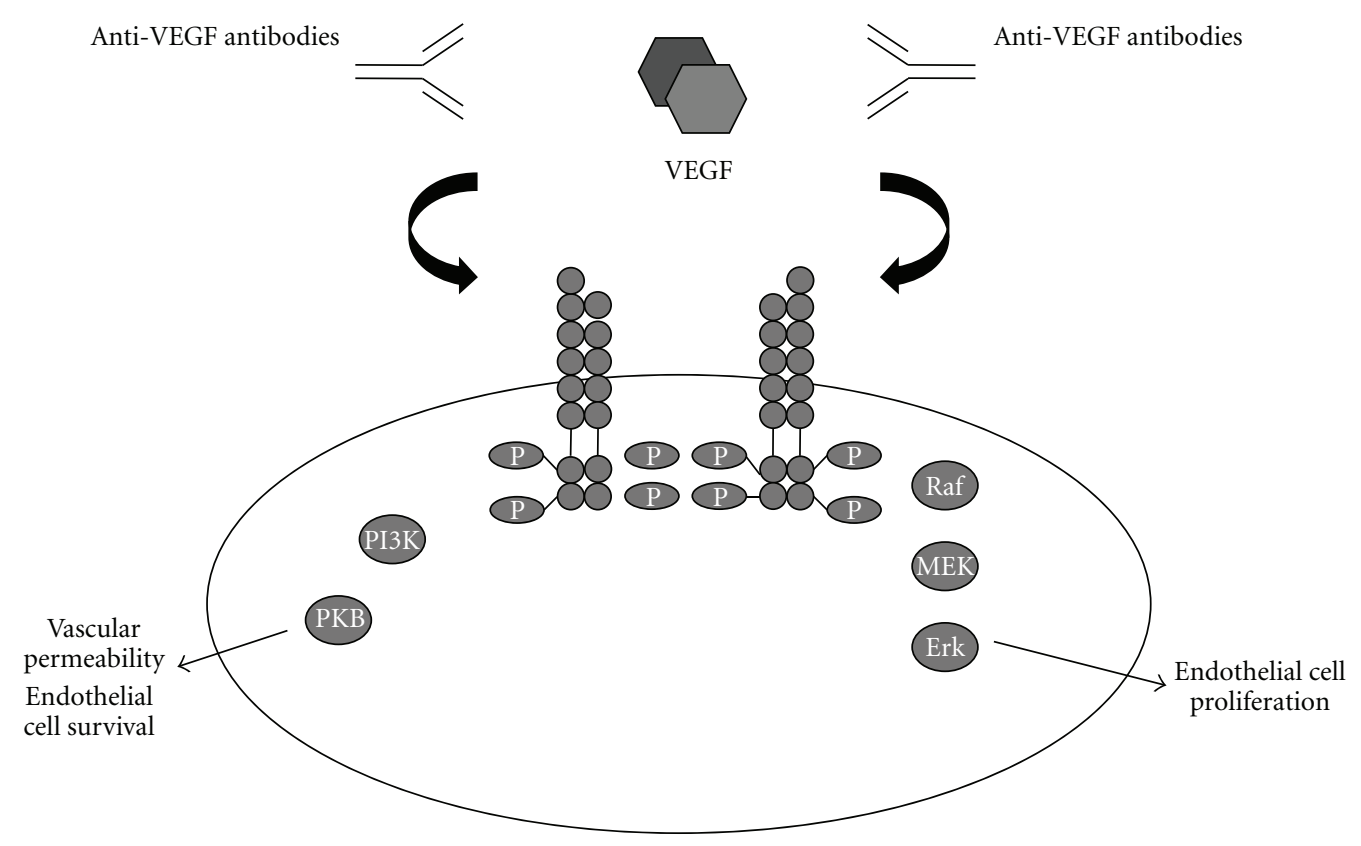

Figure 1: VEGF pathway and VEGF inhibitors.

microvessel density, and limits the infiltration of tumorassociated macrophages, but it is associated with elevated numbers of tumor-associated neutrophils [9, 10]. Selective inhibition of VEGFR2 with an anti-VEGF antibody is sufficient for effective blockade of the protumorigenic activity of VEGF in breast cancer xenografts [6]. These findings further define the complex molecular interactions in the tumor microenvironment and provide a translational tool that may be relevant to the treatment of breast cancer.

\section{Inhibition of Tumor Breast Growth}

Inhibition of VEGF binding to VEGFR2 by 2C3 has been shown to reduce tumor size both in pancreatic [11-13] and breast tumors [14]. Also the effect on tumor growth after the treatment with $\mathrm{r} 84$ in an orthotopic breast cancer model, similar to 2C3, has been evaluated. In fact, MDA-MB-231 cells $\left(5 \times 10^{6}\right)$ were injected into the mammary fat pad of nonobese diabetic NOD/SCID mice, and the therapy was initiated on day 26 after tumoral cell injection, when tumor volume reached $150 \mathrm{~mm}^{3}$. In this orthotopic human breast cancer xenograft model, the chronic treatment with $\mathrm{r} 84,2 \mathrm{C} 3$, or bevacizumab significantly reduced $(P<.001$; days 44 and 48 versus control) the tumoral growth, such that there was a $55 \%, 62 \%$, and $58 \%$ decrease, respectively, in tumor volume compared with control-treated animals. Thus, these data show that inhibition of the VEGF factor is sufficient to reduce the mass volume of MDA-MB-231-derived tumors. To determine if the effect of $\mathrm{r} 84,2 \mathrm{C} 3$, and bevacizumab on MDA-MB-231 tumor growth in vivo could be due directly to the block of VEGF activation of tumor cells, the tumor cell proliferation and migration were also evaluated in vitro, demonstrating that MDA-MB-231 cells migrated strongly toward VEGF and, consequently, this migration was blocked by the addition of 2C3, r84, or bevacizumab [8].

\section{Effect of Anti-VEGF Therapy on Tumor Environment}

VEGF is an abundant cytokine in the tumor microenvironment and is known to stimulate immune cell chemotaxis [15]; however, few studies have looked directly at the effect of inhibitors of VEGF on immune cell infiltration into tumors. It has been shown previously that 2C3 inhibits macrophage infiltration in an orthotopic pancreatic cancer model [16, 17]. In a mouse model of thyroid cancer, Salnikov et al. [18] demonstrated that treatment with bevacizumab reduced macrophage infiltration.

In nonobese diabetic (NOD)/SCID mice, inoculated with MDA-MB-231 cells, anti-VEGF therapy with 2C3, r84, or bevacizumab reduced tumor microvessel density by $50 \%$, $45 \%$, and $58 \%$, respectively [8]. Cancer and inflammation are connected by two pathways: the intrinsic and the extrinsic pathways. These two pathways converge, resulting in the activation of transcription factors, such as nuclear factor$\kappa \mathrm{B}(\mathrm{NF}-\kappa \mathrm{B})$, signal transducer and activator of transcription 3 (STAT3), and hypoxia-inducible factor $1 \alpha$ (HIF $1 \alpha)$, in tumour cells. These transcription factors modulate the production of inflammatory mediators, including cytokines and chemokines [15]. These factors recruit and activate various leukocytes. The cytokines activate the same key transcription factors in inflammatory cells, stromal cells, and tumor cells, resulting in more inflammatory mediators being produced and a cancer-related inflammatory microenvironment being generated. The mediators and cellular effectors of inflammation are important constituents of the local environment 
of tumours; in some cancers, inflammatory conditions are present before a malignant change occurs. To examine the importance of inflammatory microenvironment on tumor site, where a massive macrophage infiltration is associated, breast tumors from bevacizumab, 2C3, or r84 treatment groups were analyzed using three different macrophage markers: the mouse F4/80 antigen, a $160 \mathrm{kD}$ glycoprotein expressed by murine macrophages, and CD16+ cells, a cluster of differentiation found on the surface of natural killer cells, neutrophil polymorphonuclear leukocytes, monocytes, and macrophages.

Immunofluorescence staining of tumor sections revealed that anti-VEGF therapy with $2 \mathrm{C} 3, \mathrm{r} 84$, and bevacizumab reduced cluster of differentiation the infiltration of $\mathrm{F} 4 / 80+$ and of $\mathrm{CD} 16+$ natural killer cells, neutrophil polymorphonuclear leukocytes, monocytes and macrophages. The reduction of macrophage infiltration in the phase of treatment with 2C3 and $\mathrm{r} 84$ is presumably due to expression of VEGFR2 on tumor-associated macrophages [8]. The major findings of this study were that $2 \mathrm{C} 3, \mathrm{r} 84$, and bevacizumab effectively decrease tumor size, microvessel density, and macrophage infiltration in an orthotopic model of breast cancer. These data are consistent with previous findings [16-18] and support the concept that reducing macrophage infiltration is an important aspect of anti-VEGF therapy. In addition, these studies showed changes in other immune cell infiltrates: myeloid-derived suppressor cells (MDSCs), dendritic cells (DCs), and neutrophils, following anti-VEGF therapy. In fact, infiltrating inflammatory cells constitute a large component of the overall tumor mass [19].

\section{Anti-VEGF Therapy Leads to a Reduction in Macrophage Infiltration}

VEGF is a major chemoattractant for inflammatory cells [15], including macrophages, neutrophils, dendritic cells, myeloid-derived suppressor cells, and T-cells [20, 21].

To evaluate if inhibition of VEGF reduces angiogenesis and modulates immune cell infiltration of orthotopic breast cancer xenografts, Roland et al. [8] use preclinical models of breast cancer to compare the effect of different anti-VEGF therapies on breast cancer growth, vascular parameters, immune cell infiltration, and intratumoral cytokine levels. They confirmed that the inhibition of VEGF receptor activation resulted in changes in intratumoral levels of IL1 and CXCL1 that correlated with changes in immune cell infiltration [8].

\section{VEGF and Lung Cancer}

VEGF represents a good candidate as target of therapy also in lung cancer. In fact, $\mathrm{Ma}$ et al. constructed a plasmid encoding VEGF shRNA to knock down VEGF both in vitro and in vivo [22]. In vitro, specificity and potency of the targeting sequence were first validated in A549 lung adenocarcinoma cells by RTPCR and ELISA assays. In vivo, therapy experiments were conducted on nude mice bearing A549 xenograft tumors. The VEGF shRNA expressing plasmids were administered systemically in combination with low dose of cis-diclorodiamminoplatino (DDP) that is an antineoplastic chemotherapy agent that interferes with all phases of the cell cycle by binding to DNA through the formation of crosslinks between complementary strands. The combinated treatment of the two agents had a significantly enhanced antitumoral effect compared with the treatment with pshVEGF or DDP alone, resulting in reduction of the tumor weight by $83.13 \%$ [22]. To demonstrate that the therapeutic effects were related to downregulation of VEGF expression instead of other nonspecific reactions, the tumors were harvested for immunohistochemistry and ELISA assay to measure VEGF expression. It was analyzed the distribution of immunoreactive VEGF in the tumors and observed a general decrease of VEGF staining in the tumors belonging to the mice treated with pshVEGF, whereas the tumors belonging to the mice treated with pshHK exhibited significantly more VEGF staining. Consistently, the ELISA assay showed that pshVEGF caused significant reduction in intratumoral VEGF expression compared with pshHK [22].

These data reinforce the hypothesis that the effect of antiVEGF agents extends beyond the inhibition of angiogenesis because many immune cells express VEGFRs, including macrophages, neutrophils, MDSCs, DCs, and T-cells [23, 24]. In fact, it was shown a reduction in macrophage number in tumors from animals treated with anti-VEGF therapy with respect to the control mice [25].

\section{Concluding Remarks}

VEGF is a potent stimulator of endothelial cell survival, mitogenesis, migration, and differentiation $[1,2]$. Angiogenesis inhibitors targeting VEGF have shown antitumoral activity in preclinical and clinical trials. Several VEGF inhibitors have been approved by the US Food and Drug administration for the treatment of tumors or age-related macular degeneration $[3,4]$ (Figure 1).

Recently, in many clinical trails, angioinhibitors were also being used in combination with conventional chemotherapy. Clinical trials generally combine very low dose of chemotherapy followed by angioinhibitor therapy. Combination of angioinhibitors will need to be tested vigorously in the future, as single angioinhibitors are approved for use of cancer.

Preventive angioinhibitory therapy may also be possible in the future, because angioinhibitory therapy is generally less toxic and less susceptible to induction of acquired drug resistance.

\section{Acknowledgment}

This work was supported by a grant from the Associazione Italiana Ricerca sul Cancro (AIRC).

\section{References}

[1] J. Folkman, "Angiogenesis in cancer, vascular, rheumatoid and other disease," Nature Medicine, vol. 1, no. 1, pp. 27-31, 1995.

[2] C. R. Pradeep, E. S. Sunila, and G. Kuttan, "Expression of vascular endothelial growth factor (VEGF) and VEGF 
receptors in tumor angiogenesis and malignancies," Integrative Cancer Therapies, vol. 4, no. 4, pp. 315-321, 2005.

[3] H. F. Dvorak, "Vascular permeability factor/vascular endothelial growth factor: a critical cytokine in tumor angiogenesis and a potential target for diagnosis and therapy," Journal of Clinical Oncology, vol. 20, no. 21, pp. 4368-4380, 2002.

[4] R. Roskoski Jr., "Vascular endothelial growth factor (VEGF) signaling in tumor progression," Critical Reviews in Oncology/Hematology, vol. 62, no. 3, pp. 179-213, 2007.

[5] G. E. Konecny, Y. G. Meng, M. Untch et al., "Association between HER-2/neu and vascular endothelial growth factor expression predicts clinical outcome in primary breast cancer patients," Clinical Cancer Research, vol. 10, no. 5, pp. 17061716, 2004.

[6] K. Miller, M. Wang, J. Gralow et al., "Paclitaxel plus bevacizumab versus paclitaxel alone for metastatic breast cancer," The New England Journal of Medicine, vol. 357, no. 26, pp. 2666-2676, 2007.

[7] H. Hurwitz, L. Fehrenbacher, W. Novotny et al., "Bevacizumab plus irinotecan, fluorouracil, and leucovorin for metastatic colorectal cancer," The New England Journal of Medicine, vol. 350, no. 23, pp. 2335-2342, 2004.

[8] C. L. Roland, S. P. Dineen, K. D. Lynn et al., "Inhibition of vascular endothelial growth factor reduces angiogenesis and modulates immune cell infiltration of orthotopic breast cancer xenografts," Molecular Cancer Therapeutics, vol. 8, no. 7, pp. 1761-1771, 2009.

[9] S. M. Wilhelm, L. Adnane, P. Newell, A. Villanueva, J. M. Llovet, and M. Lynch, "Preclinical overview of sorafenib, a multikinase inhibitor that targets both Raf and VEGF and PDGF receptor tyrosine kinase signaling," Molecular Cancer Therapeutics, vol. 7, no. 10, pp. 3129-3140, 2008.

[10] M. E. Lacouture, S. Wu, C. Robert et al., "Evolving strategies for the management of hand-foot skin reaction associated with the multitargeted kinase inhibitors sorafenib and sunitinib," Oncologist, vol. 13, no. 9, pp. 1001-1011, 2008.

[11] S. P. Dineen, K. D. Lynn, S. E. Holloway et al., "Vascular endothelial growth factor receptor 2 mediates macrophage infiltration into orthotopic pancreatic tumors in mice," Cancer Research, vol. 68, no. 11, pp. 4340-4346, 2008.

[12] S. E. Holloway, A. W. Beck, L. Shivakumar, J. Shih, J. B. Fleming, and R. A. Brekken, "Selective blockade of vascular endothelial growth factor receptor 2 with an antibody against tumor-derived vascular endothelial growth factor controls the growth of human pancreatic adenocarcinoma xenografts," Annals of Surgical Oncology, vol. 13, no. 8, pp. 1145-1155, 2006.

[13] G. Korpanty, J. G. Carbon, P. A. Grayburn, J. B. Fleming, and R. A. Brekken, "Monitoring response to anticancer therapy by targeting microbubbles to tumor vasculature," Clinical Cancer Research, vol. 13, no. 1, pp. 323-330, 2007.

[14] B. Whitehurst, M. J. Flister, J. Bagaitkar et al., "Anti-VEGFA therapy reduces lymphatic vessel density and expression of VEGFR-3 in an orthotopic breast tumor model," International Journal of Cancer, vol. 121, no. 10, pp. 2181-2191, 2007.

[15] A. Mantovani, P. Allavena, A. Sica, and F. Balkwill, "Cancerrelated inflammation," Nature, vol. 454, no. 7203, pp. 436-444, 2008.

[16] S. P. Dineen, K. D. Lynn, S. E. Holloway et al., "Vascular endothelial growth factor receptor 2 mediates macrophage infiltration into orthotopic pancreatic tumors in mice," Cancer Research, vol. 68, no. 11, pp. 4340-4346, 2008.

[17] W. Zhang, S. Ran, M. Sambade, X. Huang, and P. E. Thorpe, "A monoclonal antibody that blocks VEGF binding to VEGFR2 (KDR/Flk-1) inhibits vascular expression of Flk1 and tumor growth in an orthotopic human breast cancer model," Angiogenesis, vol. 5, no. 1-2, pp. 35-44, 2002.

[18] A. V. Salnikov, N.-E. Heldin, L. B. Stuhr et al., "Inhibition of carcinoma cell-derived VEGF reduces inflammatory characteristics in xenograft carcinoma," International Journal of Cancer, vol. 119, no. 12, pp. 2795-2802, 2006.

[19] K. E. De Visser, A. Eichten, and L. M. Coussens, "Paradoxical roles of the immune system during cancer development," Nature Reviews Cancer, vol. 6, no. 1, pp. 24-37, 2006.

[20] N. Oka, A. Soeda, A. Inagaki et al., "VEGF promotes tumorigenesis and angiogenesis of human glioblastoma stem cells," Biochemical and Biophysical Research Communications, vol. 360, no. 3, pp. 553-559, 2007.

[21] Y. Takahashi, K. Yamaoka, M. Nishikawa, and Y. Takakura, "Quantitative and temporal analysis of gene silencing in tumor cells induced by small interfering RNA or short hairpin RNA expressed from plasmid vectors," Journal of Pharmaceutical Sciences, vol. 98, no. 1, pp. 74-80, 2009.

[22] J. P. Ma, Y. Yang, N. Zhang et al., "Efficient inhibition of lung cancer in murine model by plasmid-encoding VEGF short hairpin RNA in combination with low-dose DDP," Journal of Experimental \& Clinical Cancer Research, vol. 29, article 56, 2010.

[23] L. G. Presta, H. Chen, S. J. O'Connor et al., "Humanization of an anti-vascular endothelial growth factor monoclonal antibody for the therapy of solid tumors and other disorders," Cancer Research, vol. 57, no. 20, pp. 4593-4599, 1997.

[24] N. S. Templeton, D. D. Lasic, P. M. Frederik, H. H. Strey, D. D. Roberts, and G. N. Pavlakis, "Improved DNA: liposome complexes for increased systemic delivery and gene expression," Nature Biotechnology, vol. 15, no. 7, pp. 647-652, 1997.

[25] J. Tran, J. Rak, C. Sheehan et al., "Marked induction of the IAP family antiapoptotic proteins survivin and XIAP by VEGF in vascular endothelial cells," Biochemical and Biophysical Research Communications, vol. 264, no. 3, pp. 781-788, 1999. 


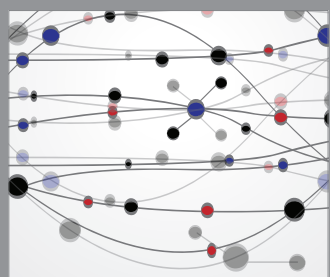

The Scientific World Journal
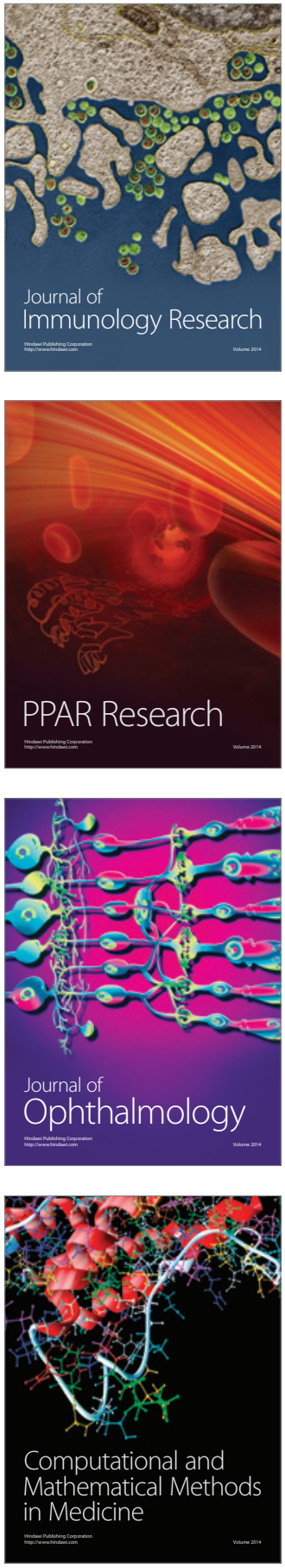

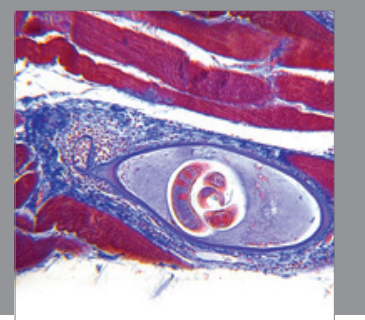

Gastroenterology

Research and Practice
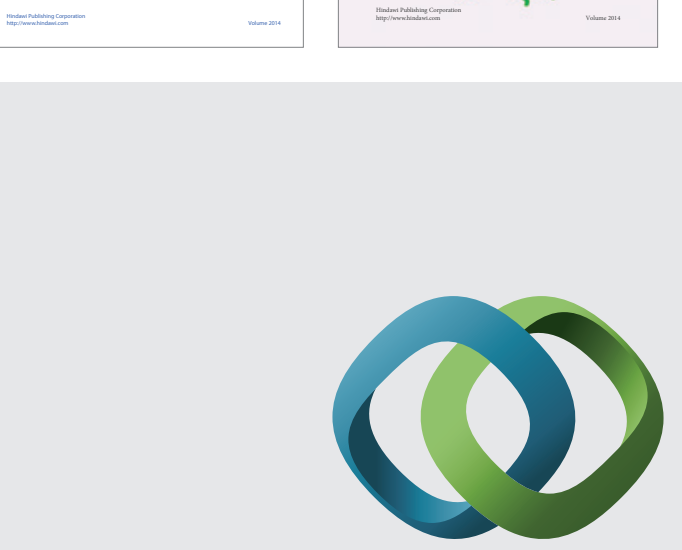

\section{Hindawi}

Submit your manuscripts at

http://www.hindawi.com
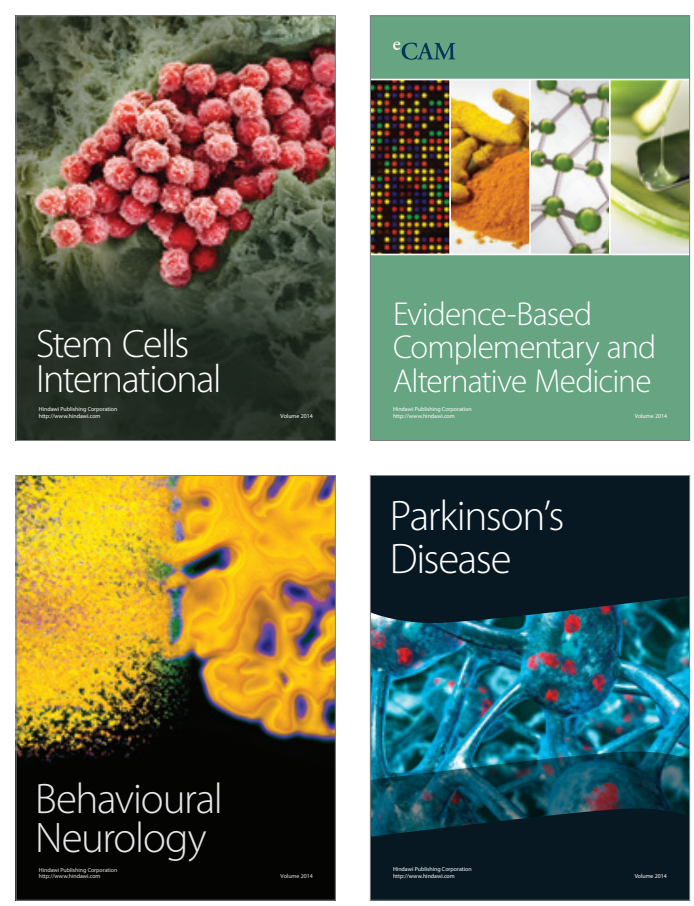

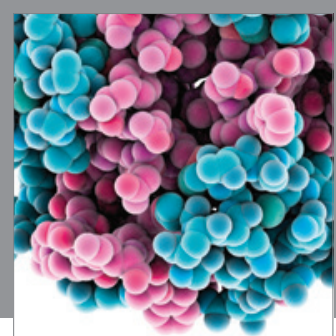

Journal of
Diabetes Research

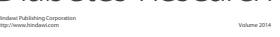

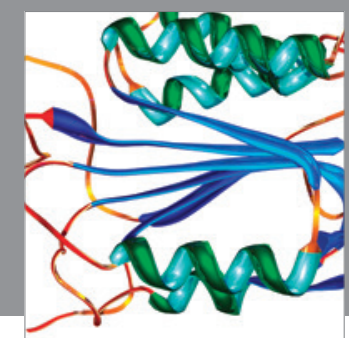

Disease Markers
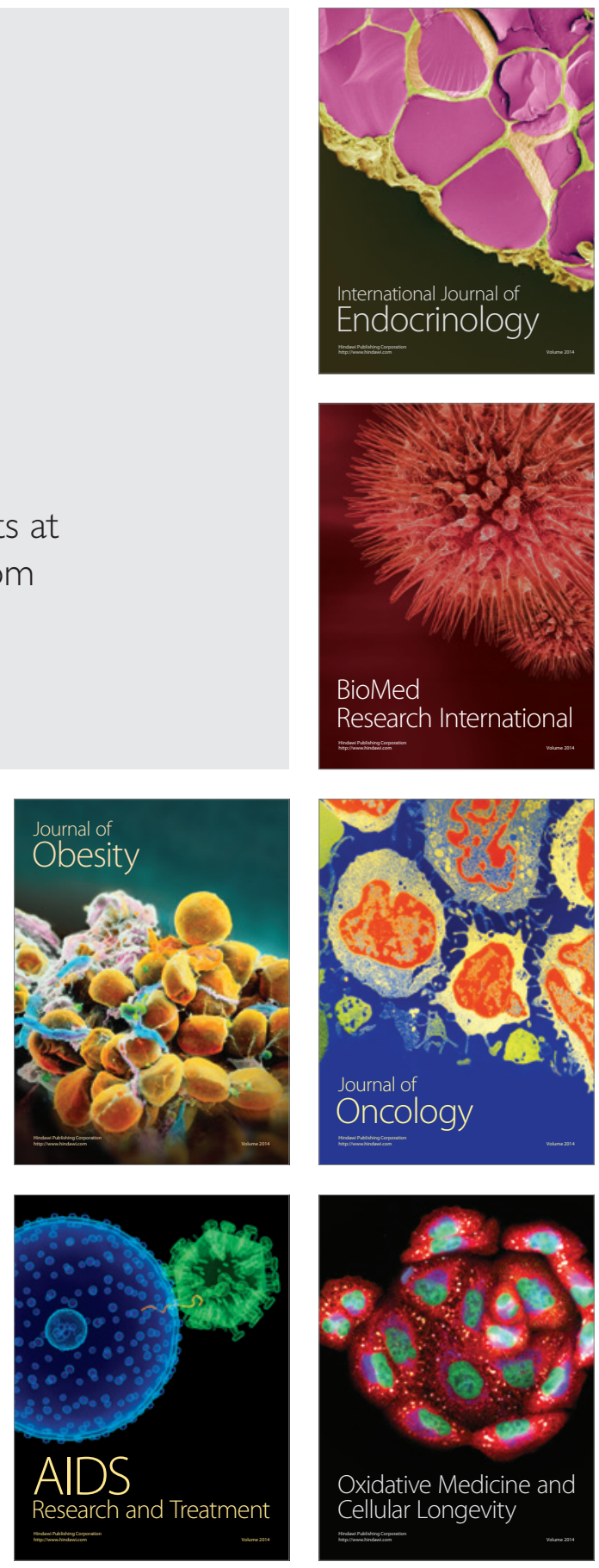